\title{
Aufzucht und Spermaproduktion bei Jungbullen unter Stationsbedingungen in Finnland
}

\author{
U. Uusisalmi und M. Kallio \\ Institut für Haustierzüchtung, Universität Helsinki, Finnland \\ G. Klautsche K \\ Sektion Tierproduktion, Universität Rostock, DDR
}

M. VARO

Institut für Haustierzüchtung, Universität Helsinki, Finnland

Eingegangen am 28.11.1972.

\section{Performance testing results and semen production in young A. I. bulls}

\author{
Unto Uusisalmi and Marja Kallio \\ University of Helsinki, Department of Animal Breeding \\ GUNTER KLautscheK \\ Sektion Tierproduktion, Universität Rostock, DDR \\ MikKo VARo \\ University of Helsinki, Department of Animal Breeding
}

\begin{abstract}
A study was made of the growth fo young A. I. bulls of Ayrshire and Finncattle breeds at performance testing stations during the first year and, subsequently, of the quantity and quality of their semen production at A. I. stations in the second year. The heritabilities of semen production characteristics, and the correlations between results of performance testing and characteristics of semen production were determined.

47 Ayrshire bulls (progeny of 10 sires) were reared at the Humppila Performance Testing Station, and 17 Finncattle bulls (progeny of 5 sires) at the Maunula Performance Testing Station, in 1967-69. The bulls were weighed at intervals of 30 days from the age of one month to the age of one year. Five different body measurements were also taken, at the age of 180 days and at the age of 365 days. Their semen production characteristics were measured at A. I. stations (Salpausselkä und Uusimaa \& Kymi) from the age of 12 months to the age of 24 months. The data on semen production for at least 8 months were required for each bull. The following characteristics were recorded: semen production per month; approved semen per month, per collection day and per ejaculate; aproved semen per collection day and per ejaculate; semen density; approved semen in percentages per month; approved semen in percentages per collection day.

The following results were obtained for the values corrected for age, number of collection days and number of ejaculations. There were no statistical differences in the
\end{abstract}


quantity and quality of semen between the two breeds (Ay and Fc). The semen production of the progeny groups of the sires differed very significantly in every individual characteristic pertaining to semen production. The individual young bulls likewise differed significantly or very significantly from one another in their semen production. It is thus possible to improve semen production by means of selection. The Ayrshire breed gave the $\mathrm{h}^{2}$ value of 0.42 for semen production per month, 0.40 for semen production per collection day, and 0.47 for semen production per ejaculate

The weight development, the growth rate and the bodily measurements of the performance testing phase correlated positively with the semen production. The young bulls that did well at the performance testing stations (weight development and growth rate good) also produced a great deal of semen during the test phase. Thus a selection based on growth results indirectly leads to improved semen production of the bull.

Aufzucht, Fütterung und Haltung üben einen grossen Einfluss auf die Fruchtbarkeit und Spermaproduktion der Zuchtbullen aus. Dies wurde mit der Einführung der künstlichen Besamung (A.I.) besonders aktuell (SchaEtz, 1963, Liebenberg und Sichting, 1971). Die Beurteilung der zweckmässigsten Aufzucht und Ernährung der Bullen wird in zunehmendem Masse von den Reproduktionsleistungen der Zuchtstiere bestimmt.

Da nur eine Mindestmenge Sperma für die Besamung der Zuchtkühe von jedem Bullen benötigt wird, ist es vorteilhaft, wenn die Jungbullen mit einer hohen Spermaproduktion einsetzen. Dadurch werden die Verweildauer und die Kosten für die Haltung der Zuchtbullen in der Besamungsstation geringer. Die Qualität und Quantität des Spermas ist, wie jede andere Leistungseigenschaft, von Erbanlage und Umwelt abhängig (EIBEL 1959). Darum ist es naheliegend, die Abhängigkeit der Spermaproduktion von der Aufzucht der Bullen zu untersuchen.

Unter modernen Zuchtbedingungen werden die Jungbullen in Stationen aufgezogen. Diese zentrale Haltung der Tiere ermöglicht eine Prüfung der Eigenleistung in den Masteigenschaften und einen Ständigen visuellen Vergleich der Körperform während der Entwichtig der Bullen. Über die Ergebnisse der Eigenleistungsprüfung der Jungbullen in Finnland berichtete Lindström (1972) ausführlich.

Ein Anliegen der Stationen ist es, die Fütterung und Haltung so zu gestalten, dass die Spermaproduktion der Zuchtbullen ständig verbessert wird. MudrA, GUNTHER und WiLKE (1969) untersuchten den Einfluss der Fütterungsintensität auf die Sexualentwicklung von Jungbullen, und fanden eine deutliche Abhängigkeit des Zeitpunktes der ersten Nutzung der Jungbullen in der Besamung von den Aufzuchtbedingungen. Sie berichten übereinstimmend mit LEIDL und Biegert (1967) und Jonova (1968), dass bei den nur Zeit üblichen Stationsbedingungen eine Zuchtbenutzung der Bullen mit einem Jahr möglich ist.

Die Fütterung und Haltung in den Stationen muss so gestaltet werden, dass die Entwicklung der Keimdrüsen nicht nur eine rechtzeitige Zuchtbenutzung der Bullen ermöglicht, sondern, auch eine hohe Spermaproduktion in der gesamten Absamperiode begründet. Die Keimdrüsen entwickeln sich im Verlaufe der. Aufzucht und bestimmen den Ablauf der Spermiogenese.

In der vorliegenden Arbeit werden die Beziehungen zwischen der Aufzucht und der Spermaproduktion bei Jungbullen unter Stationsbedingungen untersucht. 
Die Untersuchungen wurden mit Bullen der Ayrshirerasse und der Finnischen Rasse durchgeführt. Die Ayrshirebullen wurden in der Eigenleistungsprüfstation Humppilan Kasvatusasema in den Jahren 1967-69 aufgezogen und anschliessend von den Besamungsstation Salpausselkä und Uusimaa \& Kymi übernommen. In der Besamungsstation Salpausselkä konnten gleichzeitig mit den Ayrshirebullen einige Bullen der Finnischen Rasse auf ihre Samenproduktion geprüft werden. Die Bullen der Finnischen Rasse wurden in Maunula auf ihre Eigenleistung geprüft.

In die Auswertungen konnten 47 Ayrshirebullen von 10 Vätern und 17 Bullen der Finnischen Rasse von 5 Vätern einbezogen werden.

Während der Aufzucht wurden die Tiere alle 30 Tage gewogen, wobei mit 60 Tagen begonnen wurde. Ausserdem wurden die Jungbullen am 180. Tag und am 365. Tag gemessen. Es wurden der Brustumfang, die Körperlänge, die Widerristhöhe, die Kreuzbeinhöhe, der Keulenumfang, die Beckenbreite und die Brustbreite festgestellt.

Auf der Besamungsstation wurden mehrere Merkmale erfasst. Es wurde die Spermaproduktion vom 12. bis zum 24. Lebensmonat beobachtet, wobei nur Bullen zur Auswertung kamen, die mindestens 8 Monate abgesamt wurden. Die einzehnen Merkmale sind die, in der Besamung routinemässig registrierten:

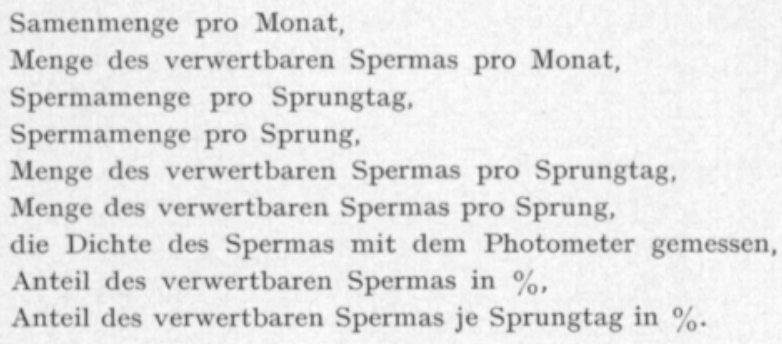

Die Auswertung erfolgt mit Hilfe mathematisch-statistischer Methoden. Es wurden Varianzanalysen (nach HARveY 1966), Korrelationanalysen und Faktorenanalysen berechnet nach den üblichen Modellen.

\section{Ergebnisse}

\section{Körperentwicklung}

Die Entwicklung der Lebendmasse der 47 Ayrshirebullen ist in der Abbildung 1 dargestellt. Mit Abschluss des 1. Lebensjahres wogen die Bullen im Durchschnitt $445 \mathrm{~kg}$. Dieser Körpermasse entspricht eine durchschnittliche tägliche Zunahme von $1228 \mathrm{~g}$ bei einer Standardabweichung von $83 \mathrm{~g}$. Die tägliche Zunahme ist für die einzelnen 30-Tage-Abschnitte in Abbildung 1 gezeigt. Der Rückgang bei der täglichen Zunahme im Alter von 150-180 Tagen beruht auf einer Umstellung der Bullen in der Station. Diese Erscheinung tritt zwar bei der Mehrzahl der Tiere auf, aber nicht bei allen. 


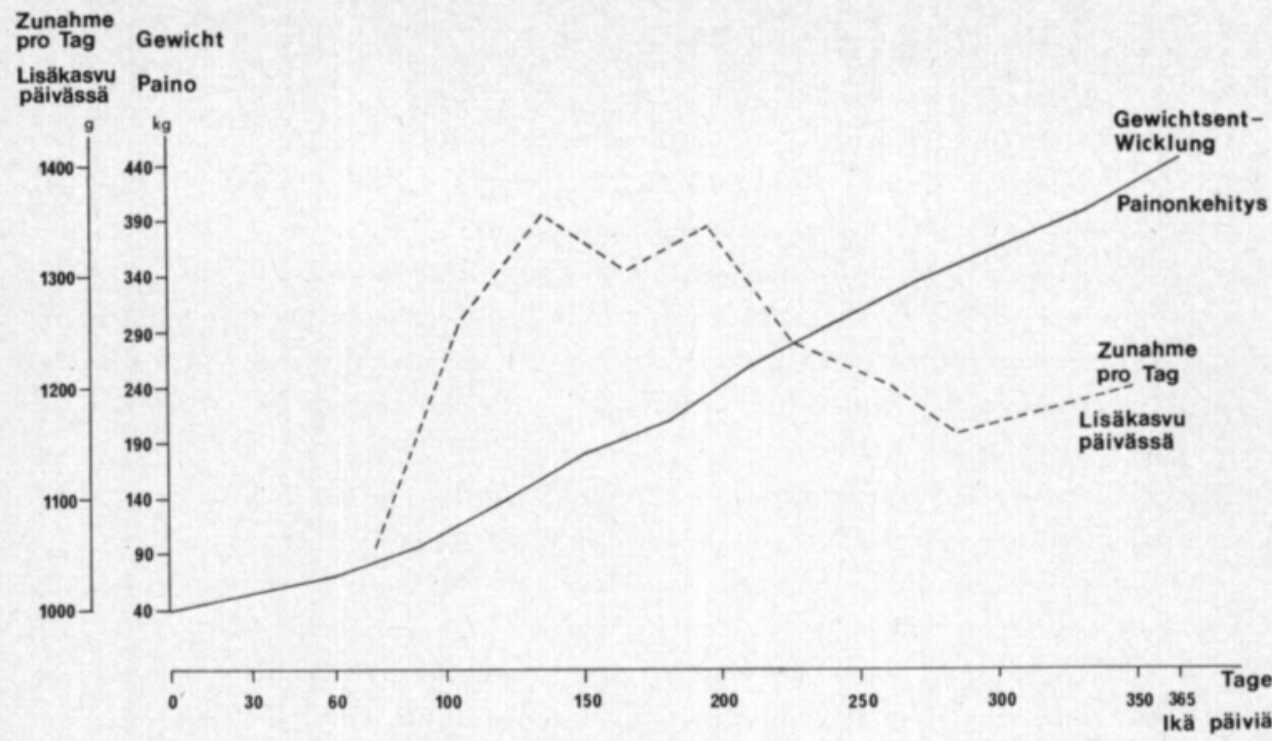

Abbildung 1. Wachstum der Bullen in der Prüfstation.

Kuva 1. Nuorien sonnien lisäkasvu ja painonkehitys yksilökokeessa.

Die Entwicklung der Tiere spegelt sich nicht nur in der Gewichtszunahme wieder, sondern wird auch durch die Körpervergrösserung deutlich. Die Körpermessungen am 180. und 385. Tag zeigen das Wachstum. In der Tabelle 1 sind die wichtigsten Masse aufgeführt.

Tabelle 1. Körpermasse der wachsenden Zuchtbullen.

\begin{tabular}{|c|c|c|c|c|}
\hline \multirow[t]{2}{*}{ Merkmal : } & \multicolumn{4}{|c|}{ Körpermasse in $\mathrm{cm}$} \\
\hline & $\overline{\mathbf{x}}$ & $\mathrm{S}$ & $\overline{\mathbf{x}}$ & $\mathrm{S}$ \\
\hline 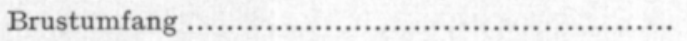 & 141 & 4.1 & 181 & 4.1 \\
\hline Körperlänge ................................................. & 108 & 3.5 & 134 & 3.4 \\
\hline 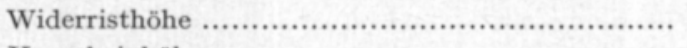 & 105 & 2.5 & 120 & 3.5 \\
\hline 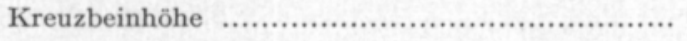 & 110 & 3.1 & 125 & 4.6 \\
\hline Keulenumfang . ............................................ & 41 & 2.5 & 52 & 1.4 \\
\hline Beckenbreite ................................................ & 35 & 1.8 & 46 & 1.9 \\
\hline 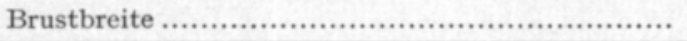 & 36 & 1.9 & 46 & 2.1 \\
\hline
\end{tabular}

Während sich die mittleren Masse im Verlaufe des Wachstums deutlich vergrössern, verändert sich die Standardabweichung wenig. Nur in der Höhe sind die Unterschiede zwischen den Tieren grösser geworden.

In Salpausselkä wurden zusammen mit den Ayrshirebullen 17 gleichartige Bullen der Finnischen Rasse aus Maunulan Kasvatusasema eingesetzt.

Mehrere qualitative und quantitative Eigenschaften der Samenproduktion wurden bestimmt. In der Tabelle 2 sind die Mittelwerte und Standardabweichungen für die zwei untersuchten Rassen getrennt ausgewiesen. 
Tabelle 2. Qualitative und quantitative Eigenschaften der Samenproduktion bei den Bullen der Finnischen Rasse und den Ayrshire

\begin{tabular}{|c|c|c|c|c|c|}
\hline Merkmal & $\bar{x}$ & $\begin{array}{c}\text { Ayrshire } \\
\text { S }\end{array}$ & $h^{2}$ & $\begin{array}{c}\text { Finnisches } \\
\bar{x}\end{array}$ & $\begin{array}{c}\text { Rind } \\
\mathrm{S}\end{array}$ \\
\hline Sperma pro Monat ..................... $\mathrm{cm}^{3}$ & 23.5 & 5.9 & 0.42 & 22.5 & 4.8 \\
\hline Verwertbares Sperma pro Monat ...... $\mathrm{cm}^{3}$ & 14.6 & 5.9 & 0.14 & 14.7 & 5.2 \\
\hline Sperma pro Sprungtag ................. $\mathrm{cm}^{3}$ & 6.0 & 1.4 & 0.40 & 5.8 & 1.2 \\
\hline Sperma pro Sprung ..................... $\mathrm{cm}^{3}$ & 2.8 & 0.6 & 0.47 & 2.8 & 0.6 \\
\hline Verwertbares Sperma pro Sprungtag $\mathrm{cm}^{3}$ & 3.7 & 1.5 & 0.10 & 3.7 & 1.3 \\
\hline Verwertbares Sperma pro Sprung ... $\mathrm{cm}^{3}$ & 1.7 & 0.7 & 0.09 & 1.8 & 0.7 \\
\hline Verwertbares Sperma in $\%$...................... & 60.0 & 19.3 & 0.02 & 64.3 & 20.4 \\
\hline Verwertbares Sperma pro Sprungtag in \% & 62.6 & 7.4 & 0.06 & 64.3 & 6.1 \\
\hline
\end{tabular}

Die Rassen unterscheiden sich in keinem Merkmal signifikant. Die Differenzen zwischen den Mittelwerten sind auffallend gering. Mit Hilfe der Varianzanalyse konnten zwischen den Nachkommengruppen und den einzelnen Bullen gesicherte Unterschiede errechnet werden. Um die einzelnen Leistungen der Bullen vergleichen zu können, wurden die Merkmalswerte nach dem Alter der Bullen bei der Absamung, nach der Zahl der Absamtage pro Jahr und nach der Zahl der Sprünge pro Jahr korrigiert. Die Sicherung der Unterschiede ist in der Tabelle 3 angegeben. Sie ist in den angegebenen Merkmalen für beide Rassen gleich.

Tabelle 3. Unterschiede zwischen den Rassen (Ay und F), den Nachkommengruppen und den Bullen bei den Merkmalen der Spermaproduktion

\begin{tabular}{|c|c|c|c|}
\hline Merkmal & $\begin{array}{c}\text { Unt } \\
\text { den } \\
\text { Rassen }\end{array}$ & $\begin{array}{l}\text { terschied zwisch } \\
\text { den } \\
\text { Nachkommen- } \\
\text { gruppen }\end{array}$ & $\begin{array}{l}\text { hen } \\
\text { den } \\
\text { Bullen }\end{array}$ \\
\hline Sperma pro Monat & - & $* * *$ & ** \\
\hline Verwertbares Sperma pro Monat ............................. $\mathrm{cm}^{3}$ & - & *** & $* * *$ \\
\hline Sperma pro Sprungtag ...................................... $\mathrm{cm}^{3}$ & - & $* * *$ & $* * *$ \\
\hline Sperma pro Sprung & - & $* * *$ & $* * *$ \\
\hline Verwertbares Sperma pro Sprungtag ..................... $\mathrm{cm}^{3}$ & - & $* * *$ & *** \\
\hline Verwertbares Sperma pro Sprung …….................. $\mathrm{cm}^{3}$ & - & *** & *** \\
\hline Fotometrische Zahl & - & $* * *$ & $* * *$ \\
\hline Verwertbares Sperma \% & - & $* * *$ & $* * *$ \\
\hline
\end{tabular}

Von den Ayrshirebullen wurden die Beziehungen zwischen der Aufzucht und der späteren Spermaproduktion ermittelt. Dabei ist die Abhängigkeit zwischen dem Jugendwachstum und den quantitativen Eigenschaften des Spermas grösser als zu den qualitativen. Die wesentlichen Korrelationswerte sind 
in der Tabelle 4 angegeben. Zwischen der Entwicklung der Jungbullen und den übrigen gemessenen Spermaeigenschaften konnten keine gesicherten Beziehungen gefunden werden.

Tabelle 4. Phänotypische Korrelation zwischen den Wachstum der Bullen und ihrer späteren Spermaproduktion

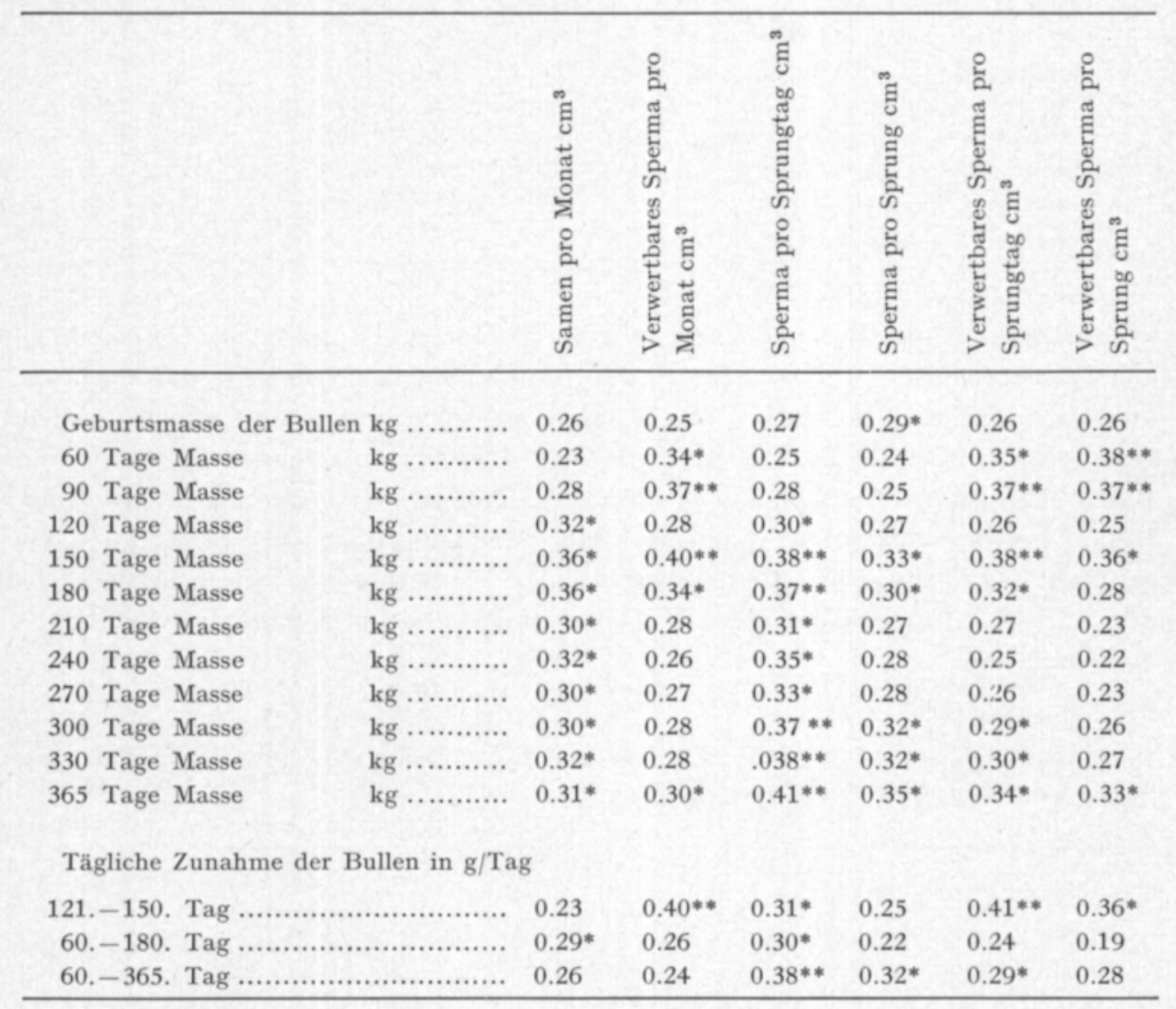

Zwischen der Körpergrösse und den Spermaeigenschaften gibt es bei den Jungbullen nur sehr geringe Zusammenhänge. Der Brustumfang, die Körperhöhe, die Körperlänge am 365. Lebenstag gemessen und die Körperbreite zeigen keinen Einfluss auf die Spermaproduktion. Die frühe Jugendentwicklung der Bullen und die quantitativen Spermaeigenschaften zeigen signifikante Zusammenhänge. Der Brustumfang, die Körperbreite und die Kreuzbeinhöhe am 180. Lebenstag gemessen sind gesichert korreliert mit der späteren Spermaerzeugung der Bullen. Die Körperlänge und Wiederristhöhe hatten in diesem Alter keinen nachweisbaren Einfluss auf die Samenproduktion. In der Tabelle 5 werden diese Zusammenhänge in Zahlen angegeben.

\section{Diskussion}

Die untersuchten Jungbullen zeigten ein normales Wachstum. Ihre Entwicklung war besser als die von MaIJALA (1969) für Ayrshire beschriebenen 
Tabelle 5. Phänotypische Korrelation zwischen der Körpergrösse der Bullen und ihrer Spermaproduktion.

\begin{tabular}{|c|c|c|c|c|c|}
\hline 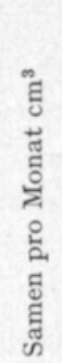 & 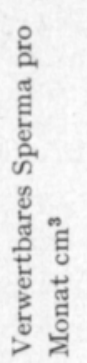 & 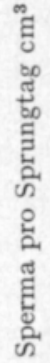 & 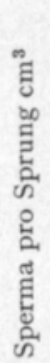 & 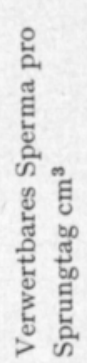 & 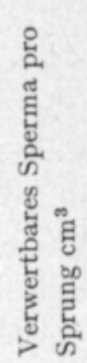 \\
\hline
\end{tabular}

Körpermasse am 180. Tag in $\mathrm{cm}$

Brustumfang .................................

Körperlänge ..............................

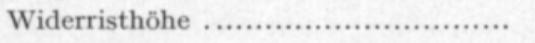

Kreuzbeinhöhe ............................

Beckenbreite ...............................

$\begin{array}{llllll}0.35^{*} & 0.39^{* *} & 0.37^{* *} & 0.36^{*} & 0.37^{* *} & 0.34^{*} \\ 0.15 & 0.13 & 0.23 & 0.27 & 0.17 & 0.21 \\ 0.28 & 0.17 & 0.29^{*} & 0.30^{*} & 0.17 & 0.18 \\ 0.38^{* *} & 0.35^{*} & 0.39^{* *} & 0.40^{* *} & 0.33^{*} & 0.34^{*} \\ 0.21 & 0.27 & 0.29^{*} & 0.31^{*} & 0.27 & 0.21 \\ 0.21 & 0.27 & 0.30 & 0.36^{*} & 0.27 & 0.22\end{array}$

Leistungen in den Aufzuchsstationen Finnlands. Die Bullen erreichten nach 12 Monaten eine vorzügliche Zuchtkondition. Die Körpermasse und die Jährlingsmasse von $445 \mathrm{~kg}$ weisen auf eine rahmige Entwicklung der Tiere. Das Wachstum ist bei jungen Tieren stärker umweltabhängig als bei älteren. LindSTRöm (1972) untersuchte für Ayrshirebullen der Prüfstation die Erblichkeit des Wachstums. Er fand folgende Werte:

\begin{tabular}{|c|c|}
\hline & $\mathrm{h}^{2}$ \\
\hline Geburtsgewicht .................. & $24 \pm 10 \%$ \\
\hline 180 Tage Gewicht .......................... & $35 \pm 13 \%$ \\
\hline 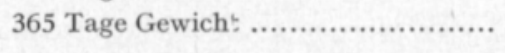 & $65 \pm 18 \%$ \\
\hline Tägliche Zunahme & \\
\hline 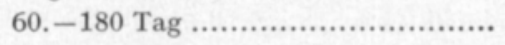 & $25 \pm 9 \%$ \\
\hline 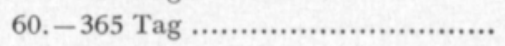 & $64 \pm 17 \%$ \\
\hline
\end{tabular}

Diese Tatsache beeinflusst auch die phänotypischen Zusammenhänge der Aufzucht der Bullen und der späteren Spermaproduktion. Erst mit der Einführung der künstlichen Besamung begann man sowohl beim Finnenrind als auch bei den Ayrshire die Bullen nach ihrer Spermaproduktion zu selektieren. In der durchgeführten Untersuchung, siehe Tabelle 2, konnten keine Unterschiede zwischen den Rassen nachgewiesen werden. Die gesicherten Unterschiede zwischen den Nachkommengruppen oder Vätern sind züchterisch von Bedeutung. Es ist möglich, die Spermaeigenschaften durch Selektion von Generation zu Generation zu verbessern. Diese Aussage wird durch die $\mathrm{h}^{2}$ Schätzwerte untermauert. Bei den Ayrshire wurde $\mathrm{h}^{2}$ für die Spermamenge pro Monat mit 0.42 für die Spermamenge je Sprungtag mit 0.40 und für die Spermamenge je Sprung mit 0.47 errechnet. Die übrigen Spermaeigenschaften wiesen 
eine niedrigere Heritabilität auf. Die gesicherten Unterschiede zwisc en den Bullen weisen gleichfalls auf erhebliche Differenzen unter den Bullen hin. Eine Auswahl der Bullen nach ihrer Spermaproduktion erscheint erfolgversprechend.

Bei zukünftigen Untersuchungen genügt es nicht, nur die Spermamenge der Bullen zu erfassen, sondern es muss die Anzahl der Tiefgefrierpellets je Bulle ausgewertet werden. Die Qualität und Quantität des Spermas ist von der Erbanlage und der Umwelt abhängig. Eine angemessene Aufzucht der Bullen bildet die Grundlage für die spätere Spermaproduktion. Sowohl eine zu reichliche Ernährung als auch eine Mangelernährung, besonders einseitige Ernährung, setzen die Geschlechtsleistung der Bullen herab. An den unter einheitlichen Bedingungen in der Station autgezogenen Bullen konnten Beziehungen zwischen Jugendwachstum und Spermaproduktion nachgewiesen werden. Aus der Entwicklung der Körpermasse und dem Brustumfang sowie der Kreuzbeinhöhe am 180 Tag gemessen, lassen sich Aussagen über die zukünftige Spermaquantität machen. Die Korrelationswerte um 0.3 deuten darauf hin, dass Bullen, die eine bessere körperliche Entwicklung während der Aufzucht zeigen, zu einer vermehrten Spermaerzeugung fähig sind.

Die Ergebnisse der Untersuchungen beweisen, dass es noch züchterische Reserven zur Steigerung von qualitativ hochwertigem Sperma gibt. Durch die Selektion der Jungbullen nach ihrem Wachstumsvermögen wird die Spermaerzeugung bei den Zuchtbullen verbessert.

\section{Zusammenfassung}

Die vorliegende Arbeit befasst sich mit der Aufzucht und Spermaproduktion bei Jungbullen. Von 47 in der Eigenleistungsprüfstation aufgezogenen finnischen Ayrshirebullen wurden lebendmasseentwicklung und Körpermasse kontrolliert. Mit 12 Monaten wurden die Bullen in die Besamungsstationen übernommen und ihre Spermaproduktion wurde registriert. Gleichzeitig mit den Ayrshirebullen wurde die Spermaerzeugung von 17 Bullen des Finnenrindes erfasst.

Die beiden Rassen unterschieden sich weder in den qualitativen noch in den quantitattiven Spermaeigenschaften.

Zwischen den Nachkommengruppen gibt es offenscihtliche Unterschiede bei den einzelnen Spermaeigenschaften. Auch die einzelnen Bullen unterscheiden sich gesichert voneinander bei der Spermaproduktion. Damit ist es dem Züchter möglich, die Spermaeigenschaften durch Selektion zu verbessern.

Zwischen dem Jugendwachstum und der Spermaproduktion wurden positive Korrelationen gefunden. Bullen, die eine bessere körperliche Entwicklung während der Aufzucht zeigten, erzeugten eine grössere Spermamenge im Untersuchungsweitraum auf der Besamungsstation. Durch die Auswahl der Jungbullen nach ihrer Eigenleistung Zunahme und Körpergrösse - wird die Spermaerzeugung bei den Zuchtbullen verbessert. 


\section{LITERATURNACHWEIS}

ErBL, K. 1959. Lehrbuch der Rinderbesamung. S. 485. Hamburg, Berlin.

HARveY, W. R. 1966. Least-squares analysis of data with unequal subclass numbers. ARS 20-28. July 1960. Reprinted with corrections April 1966. Agric. Res. Service U.S. Dept. of Agric.

Jonova, A. G. 1968. O vosproisvoditelnoj funkcii molydych byckov. Dokady TSCh A, H. 23.

Leidl, W. \& Biegert, W. 1967. Pubertät und Zuzhtreife bei Höhenfleckvieh. Tierärztl. Wochenschr. H. 18. Berlin, München.

Liebenberg, O. \& Sichting, P. 1971. Die Auswirkung von Umwelt- und Altereinflüssen auf die Spermaqualität und Untersuchungen über Möglichkeiten zur Verbesserung der Spermaeigenschaften bei Besamungsbullen. Archiv für Tierzucht 14: $339-348$.

LindströM, U. 1972. Point of view on performance testing dual purpose bulls. World Conference Ayrshire. August 2-9. Lahti.

Maijala, K. 1969. Finnish Animal Husbandry. p. 30-32. Helsinki.

MUdrA, K., Günther, \& WiLKe. 1969. Untersuchungen zur Fütterungsintensität in der Aufzucht von Jungbullen. Z. Fortpflanz., Besam. u. Aufzucht der Haustiere. S. 225239 Berlin.

- - Ueckert, P. Kemmer. 1971. Zur Entwicklung der Sexualpotenz bei Jungbullen. Archiv für Tierzucht 14: $4771-4779$.

Schaetz, F. 1963. Künstliche Besamung bei den Haustieren. S. 912. Jena. 


\title{
Nuorien keinosiemennyssonnien kasvukoetulokset ja spermantuotanto
}

\author{
Unto Uusisalmi ja Marja Kallio \\ Helsingin yliopisto, Kotieläinten jalostustieteen laitos \\ Gunther KlautscheK \\ Sektion Tierproduktion, Universität Rostock, DDR \\ MiкKо VARo \\ Helsingin yliopisto, Kotieläinten jalostustieteen laitos
}

Tarkasteltiin ayrshire- ja suomenkarjarotuisten nuorien keinosiemennyssonnien kehittymistä kasvatusasemilla niiden ensimmäisenä ikävuotena ja sen jälkeen niiden siementuotannon määrää ja laatua keinosiemennysasemilla toisena ikävuotena. Määriteltiin spermantuotantoominaisuuksien periytyvyydet sekä kasvukoetulosten ja spermantuotanto-ominausuuksien väliset vuorosuhteet.

47 ayrshiresonnia (10 isän jälkeläisryhmät) kasvatettiin Humppilan kasvatusasemalla ja 17 suomenkarjansonnia (5 isän jälkeläisryhmät) Maunulan kasvatusasemalla vuosina 1967-69: Sonnit punnittiin kuukauden iästä vuoden ikään saakka 30 päivän välein. Lisäksi niistä otettiin 5 eri ruumiinmittaa sekä 180 päivän että 365 päivän iässä. Keinosiemennysasemilla (Salpausselkä ja Uusimaa \& Kymi) mitattiin niiden spermantuotanto-ominaisuuksia 12 kk:n iästä 24 kk:n ikäån. Kultakin sonnilta vaadittiin vähintään 8 kk:n tiedot spermantuotannosta. Seuraavat ominaisuudet rekisterōitiin: siementä kk:ta kohti, hyväksyttyä siementä kk:ta kohti, siementä ottokertaa ja hyppyä kohti, hyväksyttyä siementä ottokertaa ja hyppyä kohti, siemenen tiheys, hyväksyttyä siementä kk:ssa \%, ja hyväksyttyä siementä ottokertaa kohti \%.

Tuloksiksi saatiin sonnien iän, ottokertojen ja hyppykertojen perusteella korjatuista arvoista seuraavaa: Rotujen (ay ja sk) välillä ei o|lut tilastollisia eroja siemenen määrässä ja laadussa. Isäsonnien jälkeläisryhmien spermantuotanto erosi erittäin merkitsevästi kussakin yksityisessä siemenen tuotantoa kuvaavassa ominaisuudessa. Samoin yksityiset nuoret sonnit erosivat merkitsevästi tai erittäin merkitsevästi toisistaan spermantuotannossaan. Siten on spermantuotantoa mahdollista valinnalla parantaa. Ay-rodulla saatiin $\mathrm{h}^{2}$-arviot: siementuotannolle kk:ssa 0.42 , siementuotannolle ottokertaa kohti 0.40 ja siementuotannolle hyppykertaa kohti 0.47 .

Sonnien kasvukoejakson painonkehitys, kasvukyky ja ruumiinmitat korreloituvat positiivisesti sperman tuotantoon. Nuoret sonnit, jotka kasvatusasemilla kehittyivät hyvin (painonkehitys ja lisäkasvu olivat hyvät), tuottivat myös runsaasti spermaa koejakson aikana. Näin kasvutuloksiin perustuva valinta johtaa välillisesti sonnien siementuotannon paranemiseen. 